Kaiserschnitt

\section{Schmerzintensität unterschätzt?}

— Eines der wesentlichen Problemfelder in der Therapie von Akutschmerzen scheint die Gynäkologie zu sein. Professor Hans Jürgen Gerbershagen, Klinik für Anästhesiologie, University Medical Center Utrecht, Niederlande verglich in seiner prospektiven Kohortenstudie die Daten von 179 chirurgischen Eingriffen miteinander. Mittels QUIPS-Fragebogen wurde der stärkste Schmerz im Verlauf der stationären Behandlung erfragt.

Gerade bei Kaiserschnitten, bei denen die höchste postoperative Schmerzintensität bei 6,14 auf der Skala von 0-10 lag, scheint es Verbesserungspotenzial in der Schmerztherapie zu geben. Derzeit kommen beim Kaiserschnitt primär regionalanästhetische Verfahren zum Einsatz; Opiate werden in Deutschland wegen der Stillenproblematik vermieden. Gerbershagen sprach sich für eine klare Behandlungsleitlinie aus, die Sicherheit angesichts der widersprüchlichen Empfehlungen vermittle und eine effektive Therapie gynäkologischer Schmerzen gewährleiste. Dr.Wiebke Kathmann

Trichodynie

\title{
Wenn Haarausfall schmerzt
}

— Bei vielen Krebspatienten wird therapiebedingter Haarverlust von unangenehm schmerzenden Empfindungen an der Kopfhaut begleitet. Das Phänomen der sogenannten Trichodynie ist bisher wenig erforscht. Zum ersten Mal hat ein deutsches Forscherteam nun die Qualität, Quantität und den Verlauf dieser Empfindungsstörung bei Brustkrebspatientinnen unter einer antineoplastischen Therapie evaluiert.

Wissenschaftler aus Berlin untersuchten in einer kleinen Studie mit insgesamt 31 Brustkrebspatientinnen Empfindungsstörungen auf der Kopfhaut, wie Trichodynie oder Pruritus. Mit Hilfe eines Trichodynie spezifischen Fragebogens wurden die Teilnehmerinnen in regelmäßigen Abständen nach Beginn der Chemotherapie (15 Frauen) oder der Tamoxifenbehandlung (16 Frauen) über 28 Wochen lang zu Dauer, Beginn, Intensität und Charakter der Kopfhautempfindungen befragt. In dieser Zeit kam es bei allen Frauen unter der Chemotherapie zu Symptomen einer Trichodynie; am häufigsten berichteten sie über ein Spannen der Kopfhaut („pulling“). Am stärksten war dieses 18 Tage nach Beginn der Chemotherapie, was dem Anfang des anagenen Haarausfalls entspricht. Des Weiteren traten die Missempfindungen hauptsächlich während der aktiven Phase des Haarverlustes auf. Laut der Studienautoren könnten die Symptome einer Trichodynie demnach einen Marker für den Haarverlust und/oder für perifollikuläre Entzündungsprozesse darstellen. Im Vergleich zur Chemotherapie nahmen Patientinnen, die mit Tamoxifen behandelt wurden, signifikant seltener Schmerzen auf der Kopfhaut wahr. Interessanterweise habe keine der Frauen - egal mit welcher Behandlung - elf Wochen nach Therapiebeginn noch unter Missempfindungen der Kopfhaut gelitten, berichten die Studienautoren, und das, obwohl sie zu diesem Zeitpunkt weiter behandelt worden seien. Die Wissenschaftler vermuten deshalb, dass es ein "präventives Fenster" für die Trichodynie und den Pruritus geben könnte. Bisher würde man diese Beschwerden hauptsächlich symptomatisch angehen, etwa mit nicht reizenden Shampoos.

Veronika Schlimpert

Mein Schmerz ist dein Schmerz

\section{Fehlbeurteilung durch leidende Mütter}

— Einer dänischen Studie zufolge beeinflusst der Gesundheitszustand der Mutter offenbar deren Fähigkeit, Schmerzen bei ihrem Kind objektiv zu beurteilen [Graungaard A H et al. Acta Paediatr. 2015 Sep 18. (Epub ahead of print)]. Im Rahmen einer fragebogenbasierten Querschnittstudie der Universität Kopenhagen wurde eine Untersuchung an öffentlichen Schulen durchgeführt. 131 gesunde Kinder im Alter zwischen sechs und elf Jahren sowie deren Mütter wurden unter anderem nach rezidivierenden Schmerzen, gesundheitlichen Problemen und medikamentösen Therapien befragt und ihre Antworten hinsichtlich des kindlichen Schmerzes auf Übereinstimmung überprüft. Außerdem wurde nach Zusammenhängen zwischen dem Schmerz der
Kinder und der mütterlichen Gesundheit gesucht.

Fast jedes dritte Kind berichtete über rezidivierende Schmerzen. $58 \%$ dieser Kinder gaben an, sogar wöchentlich oder häufiger unter signifikanten Beschwerden wie Kopfschmerz, Magenschmerzen oder multiplen Schmerzzuständen zu

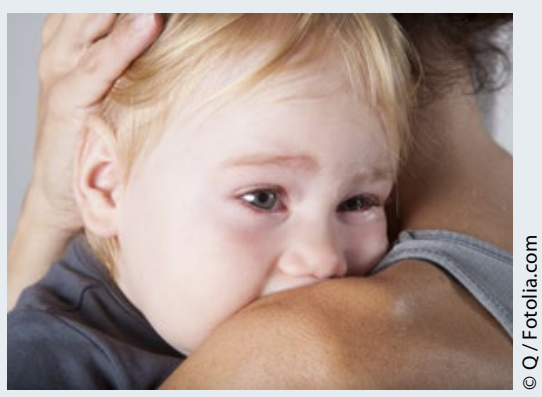

leiden. Auch $31 \%$ der Mütter berichteten über rezidivierende Schmerzen bei ihren Kindern. Wurden jedoch die Antworten von Müttern und Kindern verglichen, stimmten diese häufig nicht überein. Mütter, die selbst unter chronischen Schmerzen (meist Kopfschmerzen oder muskuloskelettaler Schmerz) litten, berichteten fünfmal so häufig über rezidivierende Schmerzzustände ihrer Kinder wie Mütter, die selbst keine Schmerzen hatten. Auch Mütter mit chronischen Krankheiten schätzten die Schmerzhäufigkeit bei ihren Kindern höher ein als gesunde Frauen. Keinen Einfluss dagegen hatten die Beschwerden der Mütter auf die Angaben der Kinder.

Weder der Bericht der Mutter noch der des Kindes allein scheinen zuverlässig zu sein, wenn es darum geht, Schmerzen bei einem Kind abzuklären.

Dr. Christine Starostzik 